\title{
Copper benchmark experiment for the testing of JEFF-3.2 nuclear data for fusion applications
}

\author{
M. Angelone ${ }^{1}$, D. Flammini ${ }^{1}$, S. Loreti ${ }^{1}$, F. Moro ${ }^{1}$, M. Pillon ${ }^{1}$, R. Villar ${ }^{1, a}$, A. Klix ${ }^{2}$, U. Fischer ${ }^{2}$, I. Kodeli ${ }^{3}$, R.L. Perel ${ }^{4}$, \\ and W. Pohorecky ${ }^{5}$ \\ ${ }^{1}$ ENEA UT-FUS, C.R. Frascati, via E. Fermi 45, 00044 Frascati (Rome), Italy \\ 2 Karlsruhe Institute of Technology (KIT), Germany \\ 3 Jožef Stefan Institute, Jamova Cesta 39, Ljubljana, Slovenia \\ 4 Racah Institute of Physics, Hebrew University of Jerusalem, Israel \\ 5 AGH University of Science and Technology, Mickiewicza 30, 30-059 Krakow, Poland
}

\begin{abstract}
A neutronics benchmark experiment on a pure Copper block (dimensions $60 \times 70 \times 70 \mathrm{~cm}^{3}$ ) aimed at testing and validating the recent nuclear data libraries for fusion applications was performed in the frame of the European Fusion Program at the $14 \mathrm{MeV}$ ENEA Frascati Neutron Generator (FNG). Reaction rates, neutron flux spectra and doses were measured using different experimental techniques (e.g. activation foils techniques, NE213 scintillator and thermoluminescent detectors). This paper first summarizes the analyses of the experiment carried-out using the MCNP5 Monte Carlo code and the European JEFF-3.2 library. Large discrepancies between calculation (C) and experiment (E) were found for the reaction rates both in the high and low neutron energy range. The analysis was complemented by sensitivity/uncertainty analyses (S/U) using the deterministic and Monte Carlo SUSD3D and MCSEN codes, respectively. The S/U analyses enabled to identify the cross sections and energy ranges which are mostly affecting the calculated responses. The largest discrepancy among the $\mathrm{C} / \mathrm{E}$ values was observed for the thermal (capture) reactions indicating severe deficiencies in the ${ }^{63,65} \mathrm{Cu}$ capture and elastic cross sections at lower rather than at high energy. Deterministic and MC codes produced similar results. The $14 \mathrm{MeV}$ copper experiment and its analysis thus calls for a revision of the JEFF-3.2 copper cross section and covariance data evaluation. A new analysis of the experiment was performed with the MCNP5 code using the revised JEFF-3.3-T2 library released by NEA and a new, not yet distributed, revised JEFF-3.2 Cu evaluation produced by KIT. A noticeable improvement of the $\mathrm{C} / \mathrm{E}$ results was obtained with both new libraries.
\end{abstract}

\section{Introduction}

Benchmarking of cross section data against integral experiments is considered since the early days of neutronics studies a fundamental step which provides feedback to the evaluators for improving both the nuclear data and the nuclear models used to produce them.

In the field of fusion neutronics, benchmark experiments were carried out in the USA (e.g. [1-3] ) already in the 80's. In the last 25 years two laboratories were performing most of the experiments (both benchmarks and mock-up) presently available at $14 \mathrm{MeV}$, namely the Fusion neutron source (FNS) in Japan (JAERI) and the Frascati neutron generator (FNG) at ENEA Frascati (Italy). The novelty of the FNG benchmark experiments, introduced since 1990s, is in the extensive use of sensitivity and uncertainty analyses applied both to the preand post-analyses of the experimental configuration using deterministic and Monte Carlo based codes.

Many materials were studied in the past both at FNG and FNS (e.g. W, Ti, $\mathrm{Cu}$, sic/SiC etc.), however not all the adjustment of the nuclear data following these experiments produced the expected data improvement. This is the case

a e-mail: maurizio.angelone@enea.it of copper. Copper is largely used in tokamaks since it is present in magnets, divertor, first wall as well as in microwave waveguides, diagnostics tools, and mirrors. It is thus important to know the copper neutron cross sections as accurately as possible to perform reliable neutronics analysis of tokamaks (e.g. ITER and DEMO). However, just a few experiments were so far reported in the literature devoted to qualify the $\mathrm{Cu}$ cross sections files at neutron energy relevant to fusion applications. These experiments (E) date back 20 years or more [4-6] and large discrepancies with calculation (C) (e.g. up to $50 \%$ for the $\mathrm{C} / \mathrm{E}$ ratio for ${ }^{197} \mathrm{Au}(\mathrm{n}, \gamma){ }^{198} \mathrm{Au}$ thermal sensor) were reported, showing the need for a revision of the used copper cross section files.

The present paper reports about a new copper benchmark experiment performed at FNG and concluded in 2015. The objective of the benchmark was the integral testing of the newly released JEFF-3.2 library [7] for copper cross sections. The used copper block $\left(60 \times 70 \times 60 \mathrm{~cm}^{3}\right)$ was the result of a pre-analysis of the experiment devoted to optimize the $\mathrm{Cu}$ block dimensions as well as the positions of the detectors [8]. The relevant neutronics quantities (e.g. reaction rates, neutron flux spectra, doses, etc.) were measured using different experimental techniques and the results 
were compared to calculation performed with the Monte Carlo code MCNP5 [9] using the fusion nuclear data library JEFF-3.2. The data analysis was complemented by sensitivity/uncertainty (S/U) analyses using deterministic and Monte Carlo based approaches as available with the SUSD3D and MCSEN codes, respectively [10-12]. This analysis allowed to identify the main contributions to the observed discrepancies between experiment and calculation and it is addressed in Sect. 3. The FNG experiment was conducted as part of a specific grant assigned by F4E, Barcelona, to the European Consortium on Nuclear Data and Experimental Techniques composed by ENEA, KIT, JSI, NPI, AGH and CCFE.

Recently, a $\mathrm{Cu}$ benchmark experiment [13] was reported from the FNS team which was a remaking of the one performed in 1995 [6] and mainly devoted to validate the $\mathrm{Cu}$ cross section of the JENDL-4.0 library [14]. The $\mathrm{C} / \mathrm{E}$ obtained are still large for all the used sensors, however, the FNS paper concluded that by using the cross sections from JEFF-3.2 for ${ }^{63} \mathrm{Cu}$ and from JENDL-4.0 for ${ }^{65} \mathrm{Cu}$, the agreement with the experiment is improved (see Fig. 13 in Ref. [13]). This was attributed to "the contemporary effect on the neutron transport of the $10 \%$ larger elastic cross section in JEFF-3.2 and to the $10 \%$ smaller capture cross section data in JENDL-4.0 in the neutron energy range between $100 \mathrm{eV}$ and $300 \mathrm{keV}$ " [13]. However, in accordance to the finding of the new FNS and the FNG experiments, revised copper cross section evaluations were produced by the NEA Data Bank, Paris, and KIT. The NEA revised Copper file is available as JEFF-3.3T2 test file.

The FNG experiment was thus re-analysed using the revised copper cross sections included in JEFF-3.3-T2 and the preliminary results (work is still ongoing) are presented in Sect. 4. Besides, the results presented in this paper include the re-analysis performed using the not yet released version of the copper cross sections developed by KIT and included in JEFF-3.3T3.

\section{2. $\mathrm{Cu}$ benchmark experiment and results}

In the new FNG copper experiment seven reaction rates (RR) were measured $\left({ }^{197} \mathrm{Au}(\mathrm{n}, 2 \mathrm{n}){ }^{196} \mathrm{Au},{ }^{93} \mathrm{Nb}(\mathrm{n}, 2 \mathrm{n})\right.$ ${ }^{92} \mathrm{Nb}^{\mathrm{m}},{ }^{27} \mathrm{Al}(\mathrm{n}, \mathrm{a}){ }^{24} \mathrm{Na},{ }^{58} \mathrm{Ni}(\mathrm{n}, \mathrm{p}){ }^{58} \mathrm{Ni},{ }^{115} \mathrm{In}\left(\mathrm{n}, \mathrm{n}^{\prime}\right){ }^{115} \mathrm{In}^{\mathrm{m}}$, $\left.{ }^{186} \mathrm{~W}(\mathrm{n}, \mathrm{g}){ }^{187} \mathrm{~W},{ }^{197} \mathrm{Au}(\mathrm{n}, \mathrm{g}){ }^{198} \mathrm{Au}\right)$. The selected nuclear reactions cover the whole energy range of interest for fusion neutronics, from $14 \mathrm{MeV}$ down to thermal energy. Eight experimental positions were selected inside the $\mathrm{Cu}$ block from $\sim 3.5 \mathrm{~cm}$ up to $\sim 57 \mathrm{~cm}$ depth, two of them were also used to measure the neutron spectrum inside the block by mean of NE-213 scintillator [15].

The RR were measured using activation foils counted by the absolute radiometric techniques by means of an absolutely calibrated HPGe detector. Experimental uncertainties range from $\pm 4 \%$ for the position nearest to the FNG target to about $\pm 8 \%$ for the foils located at the deepest positions inside the $\mathrm{Cu}$ block. Details of the experiment are reported in [16]. The experimental RR versus the penetration depth in $\mathrm{Cu}$ are shown in Fig. 1 .

The experiment was analysed with the MCNP-5 Monte Carlo code using the JEFF-3.2 cross section files for transport and the IRDFF_v1.05 dosimetric cross sections [17] for the RR calculation. The MCNP calculation rely upon a very detailed model of the experimental geometry

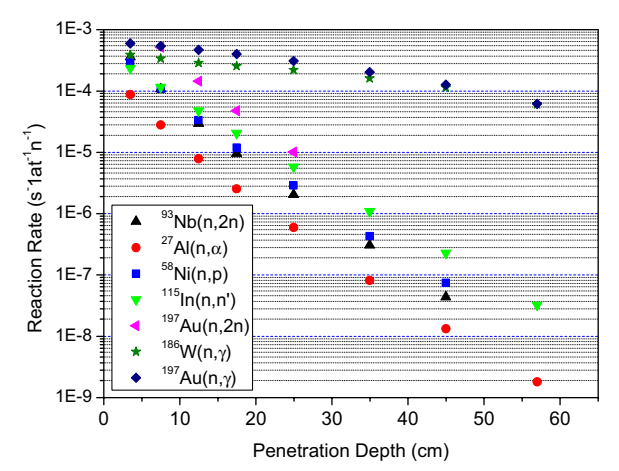

Figure 1. Experimental RR Vs. Penetration depth.
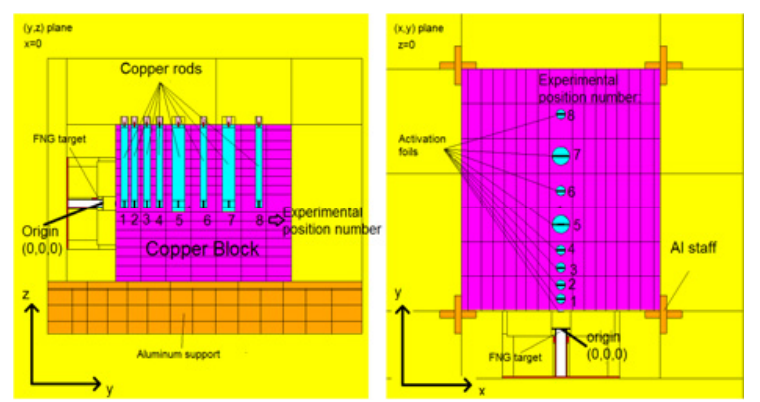

Figure 2. Lateral (left) and top (right) views of the MCNP model of the $\mathrm{Cu}$ benchmark experiment at FNG.

(Fig. 2) and of the spatial and energy distribution of the $14 \mathrm{MeV}$ neutron source. For the latter a source routine developed at ENEA and already successfully used in previous experiments was used [18]. MCNP5 was run in parallel mode using the MPI technique on the CRESCO computer cluster. The MCNP built-in weight windows (WW) variance reduction generator was adopted to generate the WW so to get statistical uncertainties $< \pm 1.5 \%$ for RR of the threshold reactions and $< \pm 3 \%$ for RR of non-threshold reactions.

An example of the $\mathrm{C} / \mathrm{E}$ ratios obtained for thermal, intermedium and fast neutron energy reactions is shown in Fig. 3. These data show large discrepancies, up to $15 \%$ for high threshold reactions, which increase up to more than $50 \%$ for thermal RR. This clearly points out deficiencies in the JEFF-3.2 Cu cross section files and calls for a new evaluation.

\section{Sensitivity and uncertainty analyses}

As already stated, a pre-analysis of the experiment was performed with sensitivity and uncertainty methods using the SUSD3D and MCSEN5 codes respectively to determine the sensitivity of the RR to the underlying cross sections and the associated uncertainties [19]. This permitted to optimise the geometry, the detector positions and the choice of the nuclear reactions to be measured according to their response in terms of sensitivity and its magnitude.

The same codes were also used for the S/U postanalyses of the experiment. Cumulative reaction rates and neutron flux spectra, their sensitivity to the cross sections, as well as the corresponding uncertainties were estimated for the selected detector positions up to $\sim 58 \mathrm{~cm}$ in the copper assembly. This permitted to interpret the results of the measurements and the calculations, to conclude 

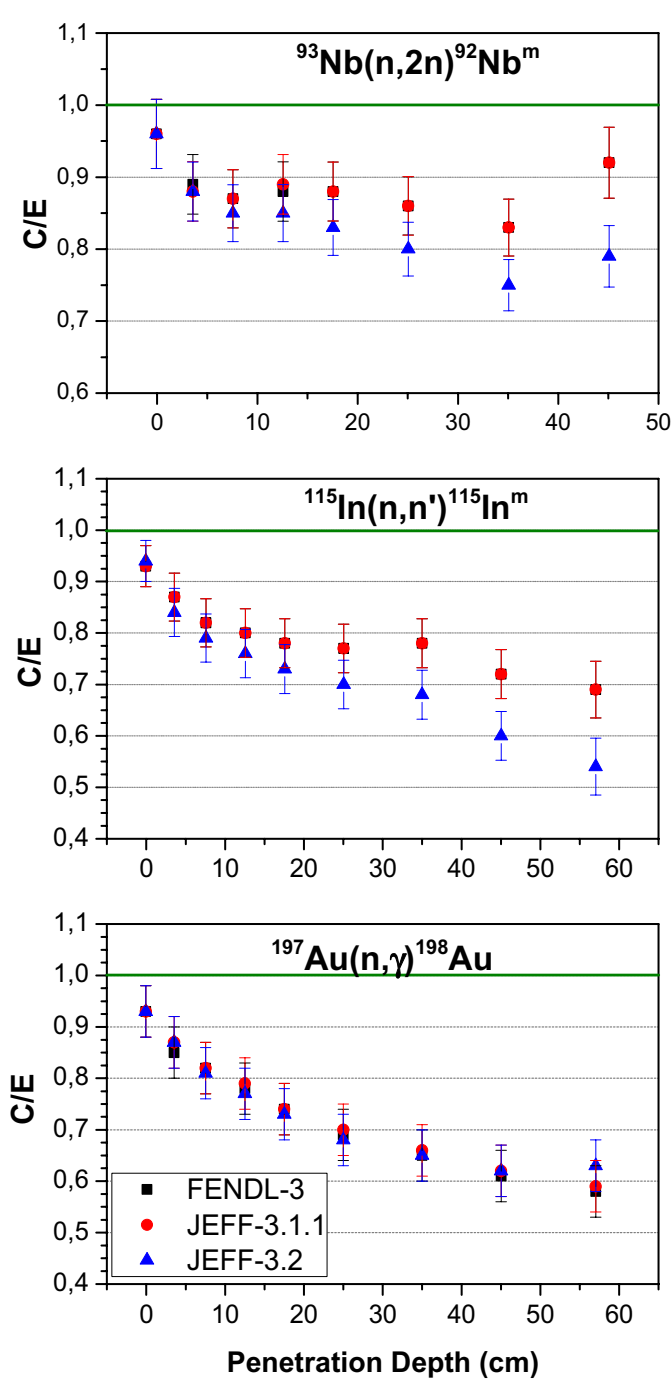

Figure 3. $\mathrm{C} / \mathrm{E}$ obtained using JEFF-3.2, JEFF-3.1.1 and FENDL-3 libraries for high, intermedium and thermal neutron energy reactions.

on the quality of the relevant nuclear cross-section data, and to estimate the uncertainties in the calculated nuclear responses and fluxes.

Several nuclear cross-section (JEFF-3.1.1 [20], JEFF3.2, FENDL-3.0 [21]) and covariance matrix evaluations (JEFF-3.2, TENDL-2013, ENDF/B-VI.8 [22,23]) were used in the analysis of the copper benchmark. Therefore, several problems were encountered in the processing of the covariance data and considerable differences among the covariance data were found. However, all files predicted large uncertainties in the calculated reaction rates and neutron spectra: up to $\sim 50 \%, 30 \%$ and 15\% using TENDL-2013, ENDF/B-VI.8 and JEFF-3.2, correspondingly.

\subsection{Results with SUSD3D code}

The objective of the sensitivity/uncertainty post-analyses was to determine the impact of the cross section uncertainties in the accuracy of the RR results and this was accomplished by using the SUSD3D code. SUSD3D requires as input quantities the direct and adjoint angular moment fluxes calculated by the Discrete Ordinates code DORT, multigroup transport cross sections and related covariance matrices for the relevant materials and reactions. The cumulative RR for the measured reactions, their sensitivity to cross-sections as well as the uncertainties were evaluated for the different detector positions, with emphasis to deep penetrations in the $\mathrm{Cu}$ block (up to $\sim 57 \mathrm{~cm}$ ). It should be mentioned that the RR calculated using FENDL-2.1 and FENDL3 transport cross sections were practically the same. The high sensitivities and uncertainties observed in the S/U pre-analysis [19] were confirmed. The uncertainties in the calculated reaction rates are particularly high for fast reactions, up to $50 \%$. Considerably different uncertainties were observed between the uncertainties based on different covariance matrices for most reactions. For fast and intermediate reactions the TENDL-2013 predictions are typically about 2 and up to $\sim 4$-times higher than those based on ENDF/B-VI.8 and JEFF3.2. According to these covariance data the reactions $(\mathrm{n}, 2 \mathrm{n}),(\mathrm{n}, \mathrm{np}),(\mathrm{n}, \mathrm{g})$, elastic and inelastic scattering on ${ }^{63,65} \mathrm{Cu}$ were found to cause the highest uncertainty which indicates the cross sections where further evaluation effort is to be concentrated. An example of the sensitivity to ${ }^{63} \mathrm{Cu}$ inelastic cross sections is shown in Fig. 4. For the thermal neutron capture reactions on ${ }^{197} \mathrm{Au},{ }^{186} \mathrm{~W}$ and ${ }^{55} \mathrm{Mn}$, consistent uncertainties between $20 \%$ and $30 \%$ were found at the deepest positions in the block using ENDF/B-VI.8 and TENDL-2013 co-variances. On the other hand, the recent JEFF-3.2 co-variances do not provide meaningful estimations for these reactions. The actually observed $\mathrm{C} / \mathrm{E}$ (dis)agreements with values as low as 0.5 partly confirm the order of magnitude of the above uncertainty predictions. This, together with the sensitivity and uncertainty results confirms the pertinence of this benchmark to contribute to the improvement of both cross section as well as covariance data evaluations. A detailed analysis of the sensitivity/uncertainty results is addressed in [24].

\subsection{Results with MCSEN5 code}

The MCSEN analysis was devoted to the assessment of sensitivities and uncertainties of the neutron flux at the two positions in the $\mathrm{Cu}$ block ( 25 and $45 \mathrm{~cm}$ depth) where the flux spectrum measurements using a NE-213 detector have been performed [15]. The sensitivities of these fluxes in 5 (coarse) energy groups (with upper group energies at $1 \mathrm{keV}, 0.1 \mathrm{MeV}, 1.0 \mathrm{MeV}, 10 \mathrm{MeV}$ and $20 \mathrm{MeV}$ ) to the cross sections of the two $\mathrm{Cu}$ isotopes, ${ }^{63} \mathrm{Cu}$ and ${ }^{65} \mathrm{Cu}$ were calculated (Fig. 5).

The neutron fluxes were shown to be most sensitive to the continuum inelastic scattering, the capture and the elastic scattering reaction cross-sections of the ${ }^{63,65} \mathrm{Cu}$ isotopes. The estimated uncertainty in the neutron spectra, based on ENDF/B-VI.8 and TENDL-2013 covariance data, is in the range $1 \%$ to $6 \%$ except for the high energy neutron flux $(\mathrm{E}>10 \mathrm{MeV})$ where the uncertainty can be $>10 \%$. It is worth to mention that very good agreement was observed between the sensitivities calculated using both SUSD3D and MCSEN codes. Relatively large differences were observed between the FENDL-2.1/3 and JEFF-3.2 cross sections causing up to $20 \%$ differences in the calculated RR, in particular for fast reactions. 


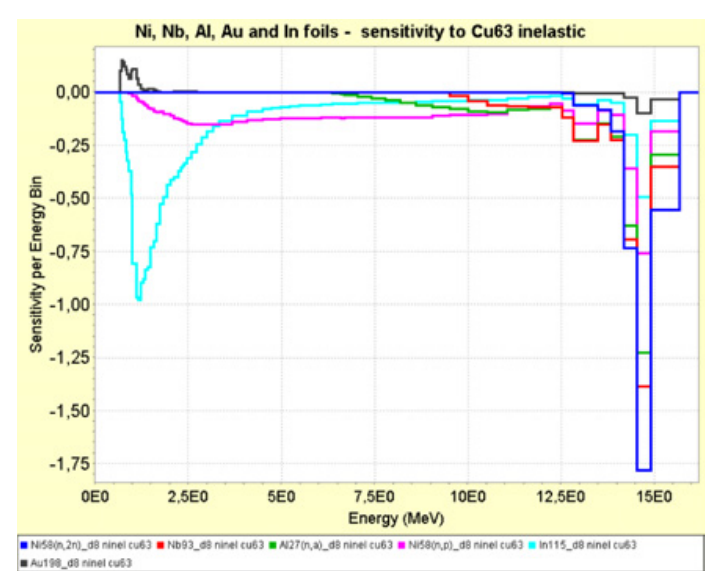

Figure 4. Example of the sensitivity profiles of the ${ }^{58} \mathrm{Ni}(\mathrm{n}, \mathrm{p})$, ${ }^{58} \mathrm{Ni}(\mathrm{n}, 2 \mathrm{n}),{ }^{93} \mathrm{Nb}\left(\mathrm{n}, \mathrm{n}^{\prime}\right),{ }^{27} \mathrm{Al}(\mathrm{n}, \alpha),{ }^{115} \mathrm{In}\left(\mathrm{n}, \mathrm{n}^{\prime}\right)$ and ${ }^{198} \mathrm{Au}(\mathrm{n}, \gamma)$ reaction rates $57 \mathrm{~cm}$ inside the FNG copper block to the ${ }^{63} \mathrm{Cu}$ inelastic cross sections.

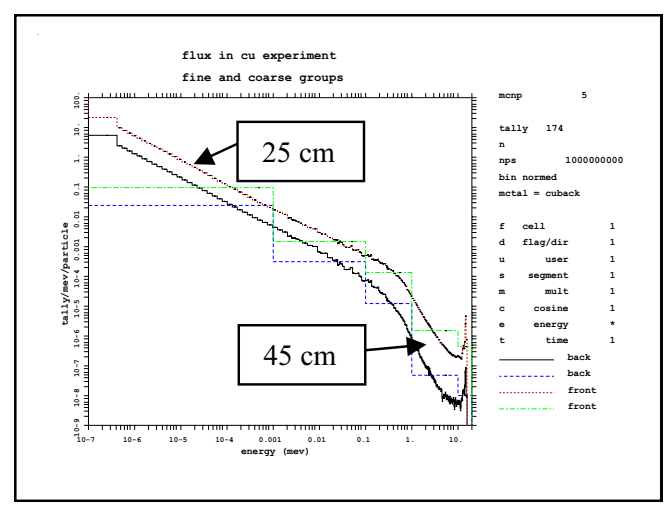

Figure 5. Calculated neutron flux spectra in the two experiental positions using MCSEN5. The picture is showing both the coarse, five groups, fluxes (step curves) used for sensitivity/uncertainty analysis as well as the 175 groups fluxes obtained from MCNP5 calculation.

\subsection{S/U analyses: Conclusions}

The reaction rates in the $\mathrm{Cu}$ block were found to be strongly sensitive to the copper nuclear cross sections. Both codes demonstrated that the most important neutron reactions were the elastic, inelastic, $(n, 2 n),(n, n p),(n, g)$ and $(n, p)$. The availability and the quality of the covariance data for transport cross sections of the uncertainty analysis was found to be an issue of concern and will require further investigations. Large differences were found among the available covariance data. Copper covariance data are available in the ENDF/B-VI.8, TENDL-2013 and -2010 and JEFF-3.2 evaluations. However, the co-variance data from the recent JEFF-3.2 evaluation of ${ }^{63,65} \mathrm{Cu}$ lead to unphysically large uncertainties at low energies below $0.1 \mathrm{MeV}$. It was thus concluded that the JEFF-3.2 covariance data of ${ }^{63,65} \mathrm{Cu}$ need to be re-evaluated. Using the above covariance matrices, the uncertainties due to cross section uncertainties were estimated to be between 10 and $50 \%(1 \sigma)$, depending on the nuclear reaction and the covariance data used. The uncertainties are particularly high for fast reactions (up to 50\%) and around $20 \%$ for the thermal reactions. On the other hand, the largest discrepancy among the measured and calculated values (C/E) was observed for the thermal (capture) reactions, suggesting problems and uncertainties in the $\mathrm{Cu}$ capture and elastic cross sections at lower energy rather than at the high energy part, as suggested by the available covariance matrices. This last result agrees with the conclusion of the FNS copper experiment [13]. Since the sensitivity to $\mathrm{Cu}$ nuclear data is high, and since the computational uncertainties are largely superior to the measurement uncertainty, the copper benchmark is likely to provide important support for the validation and improvement of copper cross-sections.

\section{Re-analysis of the FNG copper experiment using the new JEFF-3.3_T2 JEFF-3.3_T3 libraries}

The data analysis of the FNG copper experiment provides information and conclusions similar to those furnished by the 2015 FNS experiment $[13,16]$. The presently available nuclear data for copper are not satisfying and not too much improved compared to previous results, e.g. [6]. Indeed the situation was not improved since the ENDF/B$\mathrm{V}$ evaluation, regardless of the data file (JEFF, FENDL, JENDL) used for the analysis. The FNG experiment demonstrated also that, despite JEFF-3.2 was the newest available $\mathrm{Cu}$ evaluation in 2015, it provided the worst C/Es (analysis with JEFF-3.1.1 yielded better results). The above results call for a deep revision of the JEFF3.2 copper cross section files. As already said, a first adjustment of the copper cross sections was performed in Japan on the basis of the results reported in [13]. These revised ${ }^{63,65} \mathrm{Cu}$ cross sections were derived according to the FNS team proposal, that is: a) increase of the JEFF3.2 elastic cross sections of ${ }^{63} \mathrm{Cu}$ by $10 \%$ and, b) reduction of the JENDL- 4 capture cross section of ${ }^{65} \mathrm{Cu}$ by $10 \%$, in both case the cross sections were modified in the neutron energy range between $100 \mathrm{eV}$ and $300 \mathrm{keV}$. This ad-hoc modified file is not available. However, NEA provided the newly released JEFF-3.3T2 test library. The copper cross sections in JEFF-3.3T2 are a revised version of those already produced for JEFF-3.3T1 which in turn are based upon original copper data from ENDF/BVII.1 file. The main difference is in the parameters of the resonances below $6 \mathrm{keV}$ which were modified according to [25]. The FNG copper experiment was re-analysed using the MCNP5 geometry model already used in [16] and the new copper JEFF-3.3T2 library, for transport (no further modifications were introduced in the present re-analysis). The results for three reaction rates $\left({ }^{92} \mathrm{Nb}(\mathrm{n}, 2 \mathrm{n}),{ }^{115} \mathrm{In}\left(\mathrm{n}, \mathrm{n}^{\prime}\right)\right.$ and $\left.{ }^{186} \mathrm{~W}(\mathrm{n}, \gamma)\right)$ are shown in Fig. 6. In the meantime, KIT revised the JEFF-3.2 ${ }^{63,65} \mathrm{Cu}$ cross section evaluations which is planned for inclusion in the next JEFF-3.3T3 test library and is not yet released. The main modifications of the copper cross sections introduced by KIT refer to a re-evaluation of the the ${ }^{63,65} \mathrm{Cu}$ inelastic cross sections. As an example Fig. 7 shows the comparison of the new inelastic cross section for ${ }^{65} \mathrm{Cu}$ between JEFF-3.2 and the new JEFF-3.2 revision introduced by KIT. Similar results were found for ${ }^{63} \mathrm{Cu}$ inelastic scattering. The FNG copper experiment was thus also re-analysed at KIT using the same MCNP5 geometrical model used by ENEA for the above analysis with JEFF-3.3T2. The results for high threshold and intermediate neutron energy sensors are shown in Fig. 8. 


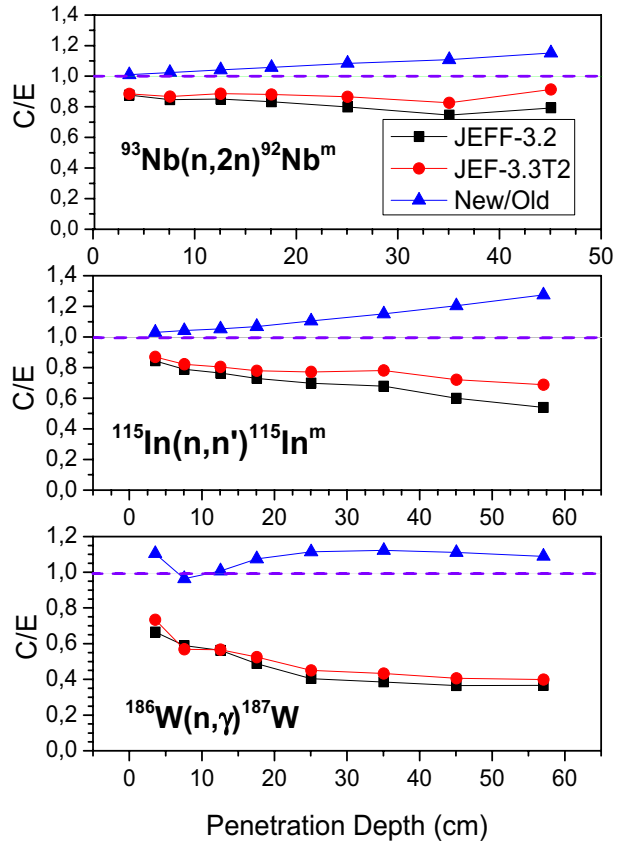

Figure 6. Calculated reaction rates using the JEFF-3.3T2 library for copper. The ratio between JEFF-3.3T2 and JEFF-3.2 results is also shown (triangles).

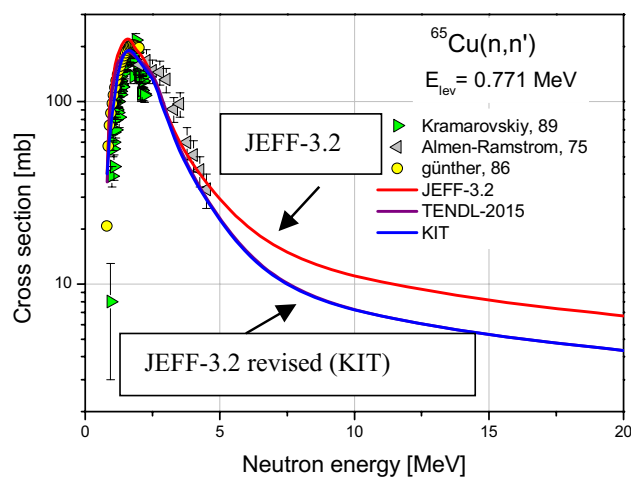

Figure 7. Revised evalaution of the JEF3.2 ${ }^{65} \mathrm{Cu}\left(\mathrm{n}, \mathrm{n}^{\prime}\right)$ reaction cross-section.

\subsection{Re-analysis using DORT and JEFF-3.3T2}

The benchmark was re-analysed using the recently released JEFF-3.3T2 cross-sections and the simplified DORT computational model. 175-group cross-sections were produced using the NJOY-99 code. The JEFF-3.3T2 data were found to increase the fast flux by up to $\sim 7$ to $8 \%$ comparing to the FENDL-3.0 results, leading to slightly improved agreement with the measurements for several fast reaction rates $\left({ }^{93} \mathrm{Nb}(\mathrm{n}, 2 \mathrm{n}),{ }^{27} \mathrm{Al}(\mathrm{n}, \alpha)\right)$, and very small overestimation of ${ }^{58} \mathrm{Ni}(\mathrm{n}, \mathrm{p})$. However, the ${ }^{115} \operatorname{In}\left(\mathrm{n}, \mathrm{n}^{\prime}\right)$, ${ }^{186} \mathrm{~W}(\mathrm{n}, \mathrm{g}),{ }^{197} \mathrm{Au}(\mathrm{n}, \mathrm{g})$ reactions remain severely underestimated similarly as using other available cross section evaluations (improvement $<5 \%$ ). According to the above finding, the $\mathrm{S} / \mathrm{U}$ analysis was not repeated since the FENDL-3 results, already discussed, apply there.

\section{Discussion and conclusion}

The results presented in this paper clearly indicate that the new copper cross section evaluation in the JEFF$3.3 \mathrm{~T} 2$ and in the not yet released JEFF-3.2 revision by
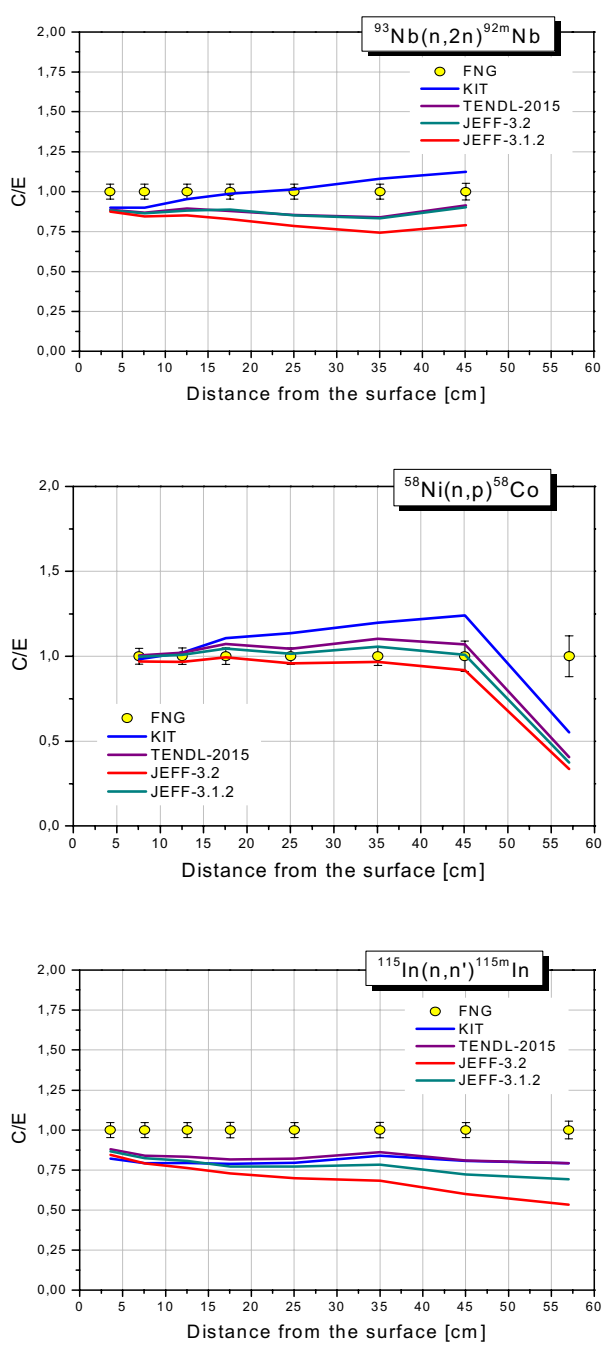

Figure 8. Results $(\mathrm{C} / \mathrm{E})$ of the re-analysis of the FNG copper experiment using the new, not yet released, JEFF-3.2. revised (LIT) evaluation provided by KIT.

KIT result in a noticeable improvement in the $\mathrm{C} / \mathrm{E}$ ratio for high threshold reactions (Figs. 6 and 7), including ${ }^{197} \mathrm{Au}(\mathrm{n}, 2 \mathrm{n}){ }^{196} \mathrm{Au}$ reaction whose threshold is around $12 \mathrm{MeV}$. This is confirmed for all the analysed RR even if for ${ }^{115} \operatorname{In}\left(\mathrm{n}, \mathrm{n}^{\prime}\right)^{115} \mathrm{In}$ reaction a clear decreasing trend in the $\mathrm{C} / \mathrm{E}$ ratio versus penetration depth is present and the $\mathrm{C} / \mathrm{E}$ ratio results about 0.75 at deep penetration. It should be mentioned that the ENEA and KIT analyses provide very similar results for the same reaction rates. The analyses performed by KIT did not yet include thermal sensors. When using JEFF-3.3T2 the ENEA calculation shows an improvement of the ${ }^{186} \mathrm{~W}(\mathrm{n}, \mathrm{g}){ }^{187} \mathrm{~W}$ reaction, the latter was providing the worst $\mathrm{C} / \mathrm{E}$ values $(<0.4)$ when using JEFF-3.2. Using JEFF-3.3T2 the C/E is increased by about 0.1 constantly all over the penetration depth. The errors on the quoted $\mathrm{C} / \mathrm{E}$ is very small as the calculated RR presents statistical errors $<2 \%$ in the deepest position. More puzzling is the situation for the ${ }^{197} \mathrm{Au}(\mathrm{n}, \gamma){ }^{198} \mathrm{Au}$ reaction. Preliminary results (not shown here), indicate a slight improvement of the results $(<5 \%)$ respect to the JEFF-3.2 case. The reason for this is to be clarified. Last, but not least, to mention that the MCNP analyses by ENEA and KIT also substantially agree with the finding of the 
DORT analysis performed by JSI (see Sect. 4.1) using multigroup representation of the JEFF-3.3T2 data.

To conclude the FNS and FNG copper benchmark experiment pointed out the need for a deep revision of the $\mathrm{Cu}$ cross section data used in JEFF-3.2 (and JENDL-4) library. Work is ongoing to improve the new copper cross section based on the two experiments. JAERI has proposed and assessment of $\mathrm{Cu}$ data by modifying the existing data for $63,65 \mathrm{Cu}$, while NEA has produced the new JEFF-3.3T2 file where parameters of resonances below $6 \mathrm{keV}$ were re-evaluated. Furthermore, KIT produced a new version of the $\mathrm{Cu}$ data evaluation to be included in the JEFF-3.3T3 release. The revision mainly affects the inelastic cross sections. Both JEFF-3.3T2 and the revised JEFF-3.2 evaluation produce a noticeable improvement for the high threshold reactions but JEFF-3.3T2 seems not to improve substantially the situation for thermal sensors as has been found by the JAERI team when modifying ad hoc the present available $\mathrm{Cu}$ cross section files. The present results thus call for further work on the copper cross section evaluations.

The work leading to this publication has been funded partially by Fusion for Energy (F4E) under the Specific Grant Agreement F4E-395-01. This publication reflects the views only of the authors and F4E cannot be held responsible for any use which may be made of the information contained therein.

\section{References}

[1] R.T. Santoro, V.C. Baker, J.M. Barnes, Nucl. Techn. 37, 274 (1978)

[2] R.T. Santoro et al., Nucl. Sci. Eng. 80, 586 (1982)

[3] R.T. Santoro et al., Nucl. Sci. Eng. 84, 260 (1983)

[4] C. Ichihara et al., J. Nucl. Sci. and Technology, 37(4), 358-367 (2000)

[5] I. Murata et al., Fus. Eng. Des. 58-59, 617-621 (2001)

[6] C. Konno et al., Fus. Eng. Des. 28, 745-752 (1995)

[7] JEFF-3.2 evaluated nuclear data library - Neutron data, OECDNEA, https://www.oecdnea.org/ dbforms/data/eva/evatapes/jeff_32/

[8] D. Flammini et al., Fus. Eng. Des. 98-99, 1964 (2015)
[9] X-5 Monte Carlo Team, MCNP - A General Monte Carlo N-Particle Transport Code, Version 5, LA-UR03-1987, LANL, Los Alamos, New Mexico, USA, (April 2003)

[10] I. Kodeli, Transactions of the American Nuclear Society 104, Hollywood, Florida, June 26-30 (2011)

[11] I. Kodeli, Nucl. Sci. Eng. 138, 45-66 (2001)

[12] R.L. Perel, Upgrading of the MCSEN sensitivity software to comply with the current standard of the MCNP-5 Monte Carlo code, Final report on Task 3.1 of the F4E Grant F4E-FPA-168.01, February 2104

[13] S. Kwon, S. Sato, M. Ohta, K. Ochiai, C. Konno, Fus. Eng. Des. 109-111, 1658 (2016)

[14] K. Shibata et al., J. Nucl. Technol. 48(1), 1 (2011)

[15] A. Klix, M. Angelone, U. Fischer, M. Pillon, Fus. Eng. Des. 109-111, 309 (2016)

[16] M. Angelone et al., Fus. Eng. Des. 109-111, 843 (2016)

[17] International Reactor Dosimetry and Fusion File (IRDFF), version v_1.05, IAEA (Vienna) Oct. 2014, https: //www-nds.iaea.org/IRDFF/

[18] A. Milocco, A. Trkov, M. Pillon, Nucl. Instrum. Meth. B-271, 6 (2012)

[19] I. Kodeli et al., Sensitivity and Uncertainty preanalysis of the FNG Cu $14 \mathrm{MeV}$ DT benchmark for fusion relevant cross sections, presented at NENE Conference, Sep. 8-11, 2015, Portoroz, SL

[20] A. Koning, R. Forrest, M. Kellett et al. (Ed.), The JEFF-3.1 Nuclear Data Library, JEFF Report 21, OECD/NEA, Paris (2006)

[21] Nuclear Data Libraries for Advanced Systems Fusion Devices (FENDL 3.0), Summary Report of the Third R. C. M. IAEA, Vienna, Austria, 6-9 Dec. (2011)

[22] A.J. Koning et al., TENDL-2013. TALYS-based evaluated nuclear data library, Dec. (2013)

[23] M.B. Chadwick et al., Nucl. Data Sheets 112, 2887 (2011)

[24] I. Kodeli et al., Fus. Eng. Des. 109-111, 1222 (2016)

[25] H. Tsuchiya et al., Nucl. Instrum. Methods A 767, 364 (2014) 